\title{
Measures of Risk for Sepsis and Mortality in Severe Burned Patients with Stress Induced Hyperglycemia
}

\author{
Albana Aleksi ${ }^{1}$, Monika Belba ${ }^{1 *}$ \\ Received: 14 February 2021 / Accepted: 07 March 2021 / Published online: 20 July 2021 \\ This article is published with open access at https://journal.astes.org.al \\ (C) The author(s) 2021. \& Copyright (C) 2021, the Albanian Society for Trauma and Emergency Surgery \\ (c) The Albanian Journal of Trauma and Emergency Surgery is an Open Access Journal. All articles are distributed under the terms of \\ the Creative Commons Attribution Non-Commercial License: http://creativecommons.org/licenses/by-nc/4.0/) which permits unrestricted \\ non-commercial use, distribution, and reproduction in any medium provided the original work is properly cited.
}

\section{Abstract}

Background: Hyperglycemia as a medical condition due to diabetes or other underlying conditions like Stress-Induced Hyperglycemia and sepsis as a life-threatening medical condition are two of the challenges faced during burn treatment.

The purpose of this study was to evaluate the risk for sepsis and mortality for the patients with critical hyperglycemic values during the disease.

Material and Methods: This is an observational retrospective cohort study conducted in the Service of Burns of the University Hospital Centre "Mother Teresa" in Tirana (UHCT), Albania from 1st January 2010 to 31st December 2014. Patients were categorized as having euglycemia (mean BG values ranging from 80-120 mg/dl), moderate hyper Sepsis was defined according to the ABA Consensus Panel Publication for Infection and Sepsis glycemia (mean BG values $<180 \mathrm{mg} / \mathrm{dl}$ ) or critical hyperglycemia (mean BG values $\geq 180 \mathrm{mg} / \mathrm{dl}$ ). to evaluate the impact of the presence of critical hyperglycemia during the disease in sepsis and mortality, we performed Relative risk, Odds ratio

Results: Those who had overall hyperglycemia (Moderate and Critical) had 2.6 times the risk for sepsis compared to those who were in the euglycemia group. Analyzing the risk of mortality in patients with overall hyperglycemia during the disease, we observed that the chance of a bad outcome was 2.7 times more likely to occur if the patient had hyperglycemia $(\mathrm{RR}=2.7)$.

Conclusions: Glucose values on admission, as one of the derangement features of burn shock, are prognostic factors in critical hyperglycemia during burn disease and have a close relationship with other outcomes (sepsis and mortality).

Keywords: Burns, Hyperglycemia, Sepsis, mortality.

\section{Introduction}

The stress response relating to critical illness refers to the systemic response to severe injury or infection. Severe burns covering more than $40 \%$ of Total Body Surface Area (TBSA) are typically followed by a period of stress, inflammation and hypermetabolism. During this period, we can observe a hyperdynamic circulatory response with in-

Original article, no submission or publication in advance or in parallel

* Corresponding author:

Asc. Prof. Dr. Monika Belba MD, Ph.D.

$\triangle$ monika.belba@fakultetimjekesise.edu.al

1 Service of Anesthesiology, Service of Burns and Plastic Surgery, University Hospital Center" Mother Teresa", Tirana, ALBANIA. creased body temperature, glycolysis proteolysis and futile substrate cycling. The hyperglycemic condition from dysregulated glucose homeostasis has been defined stress hyperglycemia. Patients with severe burns experiencing a kind of trauma undergo, among others, disturbances in glucose homeostasis, possibly leading to Stress Induced Hyperglycemia (SIH) [1]. Hyperglycemia as a medical condition due to diabetes or other underlying conditions like SIH and sepsis as a life-threatening medical condition are two of the challenges faced during burn treatment.

The purpose of this study was to evaluate the risk for sepsis and mortality for the patients with critical hyperglycemic values during the disease.

\section{Material and Methods}

This was an observational retrospective cohort study conducted in the Service of Burns of the University Hospital 
Centre "Mother Teresa" in Tirana (UHCT), Albania. UHCT is a tertiary level hospital in Albania. The study was approved by the Institutional Board of the Ministry of Health and Social Support.

We observed adult burn patients ( $\geq 20$ years old) admitted to the ICU of the Burn Service in the UHCT from 1st January 2010 to 31 st December 2014. Children and nonburn related admissions (Stevens-Johnson/toxic epidermal necrolysis, necrotizing fasciitis and chronic wounds) were excluded from the study.

Patients were categorized as having euglycemia (mean BG values ranging from $80-120 \mathrm{mg} / \mathrm{dl}$ ), moderate hyperglycemia (mean BG values $<180 \mathrm{mg} / \mathrm{dl}$ ) or critical hyperglycemia (mean BG values $\geq 180 \mathrm{mg} / \mathrm{dl}$ ) [2]. Patients with critical hyperglycemia during burn disease were treated according to the insulin regimen: basal-bolus therapy with a correctional insulin scale, with a total daily dose of 0.3-0.5 unit/ $\mathrm{kg}$ /day.

Sepsis was defined according to the ABA Consensus Panel Publication for Infection and Sepsis [3].

\section{Statistical Analysis}

The normally distributed continuous data are reported as the mean + standard deviation and analyzed using One-way ANOVA. The categorical data are expressed as frequency distributions and the z-score was used to determine whether differences existed between two population proportions. Outcome Cross tabulations are done for Sepsis and Glucose Values. In order to answer our clinical question: to evaluate the impact of presence of critical hyperglycemia during the disease in sepsis and mortality, we performed Relative risk, Odds ratio. SPSS 23 was used for statistical analysis. Values were considered significant with $\mathrm{p}<0.05$.

\section{Results}

A total of 785 patients including children were admitted to our facility over the period of study. Three hundred and forty-six (44\%) were adults. Fifty of these 346 patients died. Overall mortality was $14.5 \%$. Mean length of stay (LOS) in hospital was $14.1 \pm 17.1$ days. Mean age was $48.6 \pm 16.9$ years old. Categorization according to BMI revealed a normal distribution, peaking in the overweight group (BMI range $25-29.9 \mathrm{~kg} / \mathrm{m} 2$ ) with 154 patients $(44.5 \%$ ) (Table 1 ).

On admission there were 144 patients or $41.6 \%$ with euglycemia, 148 patients or $42.8 \%$ with moderate hyperglycemia and 54 patients or $15.6 \%$ with critical hyperglycemia. During the disease from the total number 204 were with euglycemia, 118 with moderate hyperglycemia and only 24 or $7 \%$ were with critical hyperglycemia /The last group of patients was treated with insulin according the service protocols. Observing the 24 patients who presented critical hyperglycemia during burn disease, 2 (8.3\%) of them had normal glucose values on admission; $6(25 \%)$ had moderate hyperglycemia on admission, while only $16(66.7 \%)$ of them had critical values on admission. On the other hand, observing the 54 patients with critical hyperglycemia on admission, 16 (29.6\%) had critical BG values during disease, $26(48.1 \%)$ had moderate BG values during disease and only 12 (22.3) had normal BG values during disease.

Patients belonging to the critical hyperglycemia group had a mean BSA of $39.3 \pm 23.05 \%$. There was a predominance of full-thickness burns in 19 patients (79.2\%). Sepsis occurred in 90 patients, and prevalence of sepsis in adult burn patients in our center was $26 \%$. Of the 90 patients with sepsis, 32 (36\% of 90 ) had normal BG values during burn disease, constituting $15.7 \%$ of the euglycemic group $(n=204), 44$ patients or almost $50 \%$ had moderate values

Table 1 - Demographic data for adult and elderly patients with severe burns

\begin{tabular}{|c|c|c|c|c|c|}
\hline \multirow[t]{2}{*}{ Variables } & All patients & Euglycemia & $\begin{array}{c}\text { Moderate } \\
\text { Hyperglycemia }\end{array}$ & $\begin{array}{c}\text { Critical } \\
\text { Hyperglycemia }\end{array}$ & P-value \\
\hline & $(n=346)$ & $(n=204)$ & $(n=118)$ & $(n=24)$ & \\
\hline Age, years, mean (SD) & $48.6(16.9)$ & $46.2(17)$ & $51.5(16.2)$ & $55.8(15.9)$ & 0.002 \\
\hline Glucose values on admission, mg/dl, mean (SD) & $149(57.9)$ & $129.4(39.1)$ & $162.7(48.4)$ & $248.4(100.4)$ & $<0.001$ \\
\hline \multicolumn{6}{|l|}{ Glucose values on admission \% ( $n$ ) } \\
\hline Euglycemia & $41.6(144)$ & $58.8(120)$ & $18.6(22)$ & $8.3(2)$ & \\
\hline Moderate Hyperglycemia & $42.8(148)$ & $35.3(72)$ & $59.3(70)$ & $25(6)$ & $<0.001$ \\
\hline Critical Hyperglycemia & $15.6(54)$ & $5.9(12)$ & $22(26)$ & $66.7(16)$ & \\
\hline$B S A \%$, mean (SD) & $31.8(23)$ & $26.3(18.4)$ & $39.8(27.2)$ & $39.3(23.05)$ & $<0.001$ \\
\hline Predomination of full-thickness (ves) & $78.6(272)$ & $73.5(150)$ & $87.3(103)$ & $79.2(19)$ & 0.015 \\
\hline Presence of sepsis (yes) \% (n) & $26(90)$ & $15.7(32)$ & $37.3(44)$ & $58.3(14)$ & $<0.001$ \\
\hline LOS, days, mean (SD) & $14.1(17.1)$ & $11.2(13)$ & $16.2(16.9)$ & $28.9(33.5)$ & $<0.001$ \\
\hline Mortality, deaths \% (n) & $14.5(50)$ & $8.3(17)$ & $24.6(29)$ & $16.7(4)$ & $<0.001$ \\
\hline
\end{tabular}


Table 2 - Relative risk for sepsis and mortality according glucose values categorization (during the disease)

\begin{tabular}{|c|c|c|c|c|c|}
\hline \multirow[b]{2}{*}{ Glucose values categorization } & \multicolumn{5}{|c|}{ Sepsis $(n=90)$} \\
\hline & No & Yes & Relative Risk & Odds Ratio ( $95 \%$ CI) & $p$ value \\
\hline Euglycemia $(n=204)$ & 172 & 32 & 1.0 (reference) & & \\
\hline Moderate Hyperglycemia $(n=118)$ & 74 & 44 & 2.3 & 1.6 to 3.5 & $<0.0001$ \\
\hline Critical Hyperglycemia $(n=24)$ & 10 & 14 & 3.7 & 2.3 to 5.9 & $<0.0001$ \\
\hline \multirow[t]{2}{*}{ Overall hyperglycemia } & 84 & 58 & 2.6 & 1.7 to 3.7 & $<0.0001$ \\
\hline & \multicolumn{5}{|c|}{ Mortality (Deaths) $(n=50)$} \\
\hline Glucose values categorization & No & Yes & Relative Risk & Odds Ratio ( $95 \%$ CI) & $p$ value \\
\hline Euglycemia $(n=204)$ & 187 & 17 & 1.0 (reference) & & \\
\hline Moderate $H_{y p e r g l y c e m i a ~}(n=118)$ & 89 & 29 & 2.9 & 1.6 to 5.1 & 0.0002 \\
\hline Critical Hyperglycemia $(n=24)$ & 20 & 4 & 2 & 0.7 to 5.4 & 0.1759 \\
\hline Overall hyperglycemia & 109 & 33 & 2.7 & 1.6 to 4.8 & 0.0002 \\
\hline
\end{tabular}

during burn disease constituting $37.3 \%$ of the moderate group $(n=118)$ and 14 patients or $15 \%$ had critical values during burn disease constituting $58.3 \%$ of the critical group.

Analyzing outcomes (sepsis and mortality) in patients with overall hyperglycemia during the disease (84 patients), we calculated Relative Risk and Odds Ratio (Table 2).

Those who had overall hyperglycemia (Moderate and Critical) had 2.6 times the risk for sepsis compared to those who were in euglycemia group. The risk for sepsis of patients with critical hyperglycemia among those with overall hyperglycemia was 3.7 times as high as the risk of sepsis among those who did not have critical hyperglycemia.

The risk for mortality for patients with overall hyperglycemia was 2.7 times the risk for mortality to the patients with euglycemia during the disease. It was the same as for patients with moderate hyperglycemia and with critical hyperglycemia (No statistical significance).

\section{Discussion}

Homeostasis is the maintenance of a stable internal environment in an organism through careful regulation of many parameters, including maintaining blood glucose levels within the narrow range of $60-120 \mathrm{mg} / \mathrm{dl}$. Patients with severe burns experience one of the most severe disruptions of homeostasis. Clinical situations associated with glucose homeostasis disorders are previous diagnosis of diabetes, $\mathrm{SIH}$ and relative deficiency of insulin in the preoperative period. Based on many studies, hyperglycemia might have different biological or clinical implications and has been associated with an increased risk of adverse outcomes [4, 5, 6].

A significant proportion of mortality and morbidity in severely burned patients with SIH is attributable to hypermetabolic response [7, 8]. Results of various studies researching altered metabolism and the role of insulin in the treatment of burn patients indicate that improvement in BG levels results in lower rates of hospital complications and mortality $[9,10,11,12,13,14]$.

In this clinical and epidemiological study, we have tried to assess the effect of critical hyperglycemia on admission as a real forerunner on outcome (sepsis and mortality) in adult patients with severe burns.

Analyzing sepsis and its distribution in the three groups of patients, we noticed that sepsis $(n=90)$ was present in 32 patients $(35.6 \%)$ in the euglycemic group, in 44 patients $(48.8 \%)$ in the moderate hyperglycemic group and in $14(15.6 \%)$ in the critical hyperglycemic group. We noticed that the majority of patients with sepsis (58 out of 90 patients or $64.4 \%$ ) had hyperglycemia during disease. While analyzing sepsis within each group, we noticed differences. In the euglycemic group composed of 204 patients, there were $32(15.6 \%)$ patients diagnosed with sepsis; in the moderate hyperglycemic group of 118 patients, 44 (37.2\%) had sepsis; and in the critical hyperglycemic group of 24, 14 $(58.2 \%)$ had sepsis. We noticed that sepsis was present in the majority of patients with critical hyperglycemia, which is consistent with recent studies [7].

Analyzing sepsis and the risk of developing it in patients with critical hyperglycemia during the disease, we observed a relative risk of 3.7 , meaning that the chance of sepsis is $270 \%$ higher if the patient presents with critical hyperglycemia during the disease. An OR of 1.7 indicates that the odds for sepsis are higher in the group with overall hyperglycemia (Moderate and critical) (Odds Ratio >1). In absolute values that would mean that the incidence of sepsis is $30 \%$ smaller in the group without hyperglycemia during the disease.

Analyzing mortality, we noticed that out of 50 deaths in total, 17 (34\%) were patients with euglycemia, 29 (58\%) were patients with moderate hyperglycemia and only $4(8 \%)$ were in the critical hyperglycemic group. If we look at mor- 
tality as the proportion of deaths within groups, we notice that the highest mortality $24.6 \%$ (29 deaths) was in the moderate hyperglycemic group, followed by $16.7 \%$ (4 deaths) in the critical hyperglycemic group and the lowest values, $8.3 \%$ (17 deaths), in the euglycemic group.

Analyzing the risk of mortality in patients with overall hyperglycemia during the disease, we observed that the chance of a bad outcome was 2.7 times more likely to occur if the patient had hyperglycemia $(R R=2.7)$. With an $O R$ of 2.0 for patients with critical hyperglycemia and 2.9 for patients with moderate hyperglycemia, we concluded that the odds for a bad outcome were higher but similar within groups $(\mathrm{OR}>1)$. On the other side we have noticed that there was no statistical significance for patients in critical hyperglycemia group because these patients were treated with insulin which can serve as a therapeutic agent in patients with burns and pays an important role in prognosis of severe burn patients

\section{Conclusions}

Glucose values on admission, as one of the derangement features of burn shock, are prognostic factors in critical hyperglycemia during burn disease, and have a close relationship with other outcomes (sepsis and mortality). Testing for critical hyperglycemia in the first 24 hours of admission should be done with caution to predict a probable critical hyperglycemic event during the course of burn disease.

COI Statement: This paper has not been submitted in parallel. A part of this study is presented as oral presentation in the Conference: Balkan States Anesthesia Days: Currents Topics in Anesthesia and Intensive Care 15-18 Maj, 2015 Tirana, Albania Website: http://arud2015.org

It has not been published nor submitted for consideration beforehand.

This research received no specific grant from any funding agency in the public, commercial, or nonprofit sectors. There are no relevant or minor financial relationships from authors, their relatives or next of kin with external companies.

\section{Conflict of Interest Statement}

There is no conflict of interest

\section{Authorship Contribution}

The first author has done the conception or design of the work and data collection and analysis.

The second author has done the data analysis and interpretation as well as drafting the article and critical revision of the article.

\section{References}

1. Mizock BA: Alterations in fuel metabolism in critical illness: hyperglycaemia. Best Pract Res Clin Endocrinol Metab, 15: 533-551, 2001.

2. Van den Berghe G, Wouters P, Weekers F, Verwaest Ch et al.: Intensive insulin therapy in critically ill patients. $\mathrm{N}$ Engl $\mathrm{J}$ Med, 345: 1359-1367, 2001.

3. Greenhalgh DG, Saffle JR, Holmes JH 4th et al.: American Burn Association Consensus Conference on Burn Sepsis and Infection Group. J Burn Care Res, 28: 776-90, 2007.

4. Egi M, Bellomo R, Stachowski E et al.: Blood glucose concentration and outcome of critical illness: the impact of diabetes. Critical Care Medicine, 36(8): 2249-55, 2008.

5. Duncan AE: Hyperglycaemia and Perioperative Glucose Management. Curr Pharm Des, 18(38): 6195-6203, 2012.

6. Duncan AE, Abd-Elsayed A, Maheshwari A, Xu M et al.: Role of intraoperative and postoperative blood glucose concentrations in predicting outcomes after cardiac surgery. Anesthesiology, 112(4): 860-71, 2010.

7. Meccott GA, Al -Mousawi AM, Gauglitz GG, Herndon DN, Jeschke MG: The role of hyperglycaemia in burned patients: Evidence-based studies. Shock, 33(1): 5-13, 2010.

8. Cree MG, Aarsland A, Herndon DN et al.: Role of fat metabolism in burn trauma-induced skeletal muscle insulin resistance. Crit Care Med, 35: S476-83, 2007.

9. Pidcoke HF, Wade Ch E, Wolf SE: Insulin and the burned patient. Crit Care Med, 35 (9) (Suppl.): S524-S530, 2007.

10. Gauglitz GG, Halder S, Boehning DF, Kulp GA et al.: Postburn hepatic insulin resistance is associated with endoplasmic reticulum (ER) stress. Shock, 33: 299-305, 2010.

11. Jeschke MG, Kraft R, Song J, Gauglitz GG et al.: Insulin protects against hepatic damage postburn. Mol Med, 17: 516$522,2011$.

12. Pereira CT, Murphy KD, Herndon DN: Altering metabolism. J Burn Care Rehabil, 26: 194-199, 2005.

13. Gore DC, Chinkes D, Heggers J, Herndon DN et al.: Association of hyperglycaemia with increased mortality after severe burn injury. J Trauma, 51(3): 540-544, 2001.

14. Umpierrez GE, Isaacs SD, Bazargan N, You X et al.: Hyperglycaemia: an independent marker of in-hospital mortality in patients with undiagnosed diabetes. J Clin Endocrinol Metab, 87(3): 978-82, 2002. 